\title{
Exogenous lipoid pneumonia associated with oil-based oral and nasal products
}

\author{
Sabrina H.M. Yeung MD HBSc, Lianne E. Rotin MD PhD, Kevin Singh MD HBSc, Robert Wu MD MSc, \\ Matthew B. Stanbrook MD PhD
}

Cite as: CMAJ 2021 October 12;193:E1568-71. doi: 10.1503/cmaj.210439

A 30-year-old man presented to our emergency department with left-sided chest pain. His medical history included irritable bowel syndrome, recurrent sinusitis and persistent symptoms of dry mouth, which started after a remote tonsillectomy. He had also had a $5 \mathrm{~kg}$ weight loss over the preceding year, which he attributed to dietary modifications. The patient was originally from Poland, but had been living in Toronto as a student for most of the past 2 years. He was a lifelong nonsmoker, rarely drank alcohol and did not use any recreational drugs or electronic cigarettes. About 2 weeks before presentation, at a medical facility in Poland, pneumonia was diagnosed based on findings from chest radiography and computed tomography (CT). He was treated with clarithromycin for 14 days. An echocardiogram at that time had shown a small pericardial effusion.

Ongoing symptoms of chest pain and dyspnea led to several emergency department visits and hospital admissions from August to November 2020 (Figure 1). Initially, presumed pericarditis secondary to pneumonia was diagnosed on the basis of the positional and pleuritic nature of the chest pain, and he was treated with high-dose ibuprofen and colchicine. The persistence of his symptoms led to multiple subsequent chest radiographs and CT scans. Although these ruled out pulmonary embolism, they showed persistent bilateral consolidations, nodules and ground-glass opacities in the right middle lobe, right lower lobe and left lower lobe of the lung that were largely unchanged over time (Figure 2 and Figure 3).

Before the patient was first admitted to our hospital, he was prescribed amoxicillin for 7 days for nonresolving pneumonia. Subsequently, we obtained bronchoalveolar lavage results from a bronchoscopy done in Poland that showed growth of Pseudomonas aeruginosa and Serratia marcescens. We therefore broadened antimicrobial coverage to ciprofloxacin for 14 days, which resulted in reduced dyspnea but no change in the patient's chest pain. Throughout these encounters, the patient's highest recorded temperature was $39.6^{\circ} \mathrm{C}$, his respiratory rate was 28 breaths/minute and he never required supplemental oxygen. His fever and tachypnea resolved before discharge. His blood work was unremarkable except for a mild normocytic anemia, including negative autoantibody tests for Sjögren syndrome and other connective tissue diseases.

\section{Key points}

- Nonresolving pneumonia has been defined as pneumonia with an absence of clinical improvement, clinical worsening or absence of radiographic resolution within 12 weeks after adequate antibiotic therapy.

- Exogenous lipoid pneumonia is a rare cause of nonresolving pneumonia, but should be considered in the differential diagnosis.

- A thorough exposure history, including inhalation or aspiration of fatty substances, helps to diagnose exogenous lipoid pneumonia.

- Treatment of superimposed bacterial infections with antibiotics may provide temporary symptomatic improvement.

We performed a repeat bronchoscopy with bronchoalveolar lavage, which showed yellow mucoid secretions arising from the right lung. Cytopathology was normal and cultures showed only commensal flora with no evidence of other bacteria, acid-fast bacilli, fungi or viruses, including SARS-CoV-2. We also considered connective tissue disease in the differential diagnosis, but all investigations for autoimmune diseases were unremarkable. After a multidisciplinary team discussion of his case, the patient underwent a CT-guided transthoracic lung biopsy and was discharged home. One week later, he returned to the emergency department with recurrent fever and a new patchy left lower lobe opacity that we treated with a 6-week course of amoxicillin-clavulanic acid. Pulmonary function tests done 4 weeks after the lung biopsy showed a mild restrictive pattern (total lung capacity $79 \%$ predicted, forced vital capacity $58 \%$ predicted, diffusion capacity $83 \%$ predicted).

Pathology results from the lung biopsy showed an amorphous acellular substance with a foreign-body giant cell reaction, but further identification was not possible (Figure 4). Further staining excluded amyloid, mucin, calcium, fungus and acid-fast bacilli. This prompted us to take a detailed history of environmental exposures to vaping liquids, silica, asbestos, talc and numerous other organic and inorganic materials. The patient denied all of these exposures. However, he disclosed 
Presented in Poland

- Pneumonia diagnosed

- Treated with clarithromycin,

with improvement

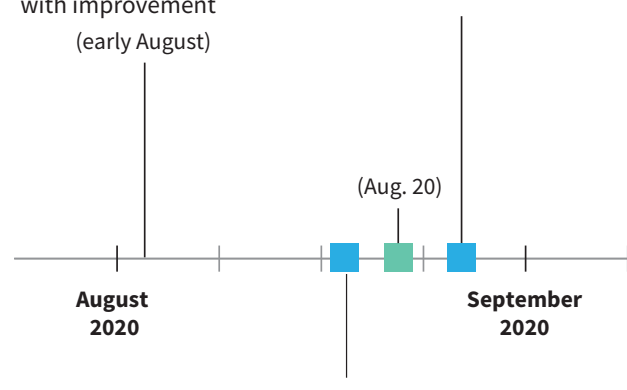

First presentation in Canada for chest pain - Pneumonia with secondary pericarditis diagnosed

- Treated with ibuprofen and colchicine (Aug. 16)
Emergency department visit

General internal medicine outpatient visit

Hospital admission

Infectious disease outpatient visit

Respirology outpatient visit
Ongoing Referred to respirology chest pain and dyspnea

(Sept. 14) for bronchoscopy for persistent consolidations (Oct. 9)

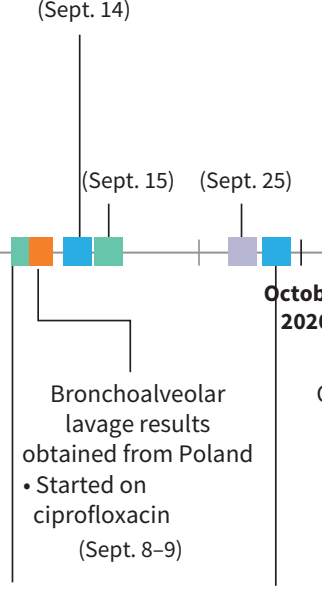

Sent for CT pulmonary angiogram and admitted

(Sept. 8)
Myalgias/arthralgias likely related to ciprofloxacin (Sept. 30)
Admitted for

expedited work-up

- Bronchoscopy and bronchoalveolar lavage - CT-guided biopsy

(Oct. 9-15)

Figure 1: Timeline of medical encounters. Note: $\mathrm{CT}$ = computed tomography.

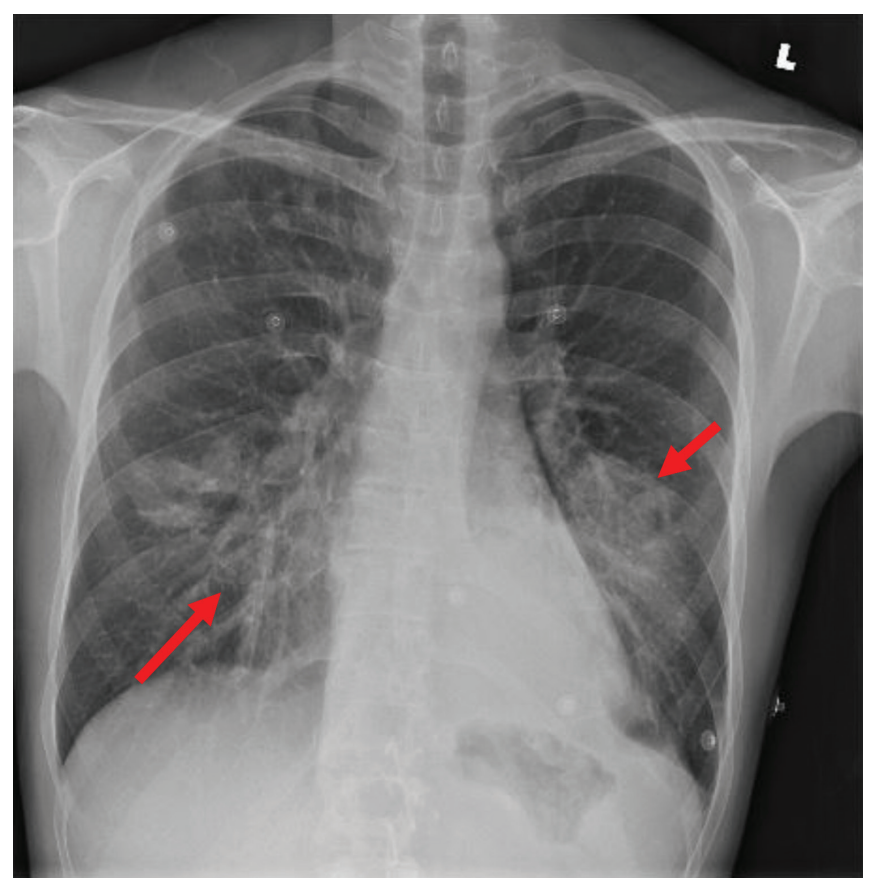

Figure 2: Posterior-anterior view chest radiograph from a 30-year-old man, showing air space opacities in the right lower lobe (long arrow) and adjacent to the left heart border (short arrow).

that he had been swishing and spitting flax seed oil for the past 12 years to help with the symptoms of dry mouth that he had been experiencing since his tonsillectomy. Recently, he noticed that he had been coughing after this process. He also

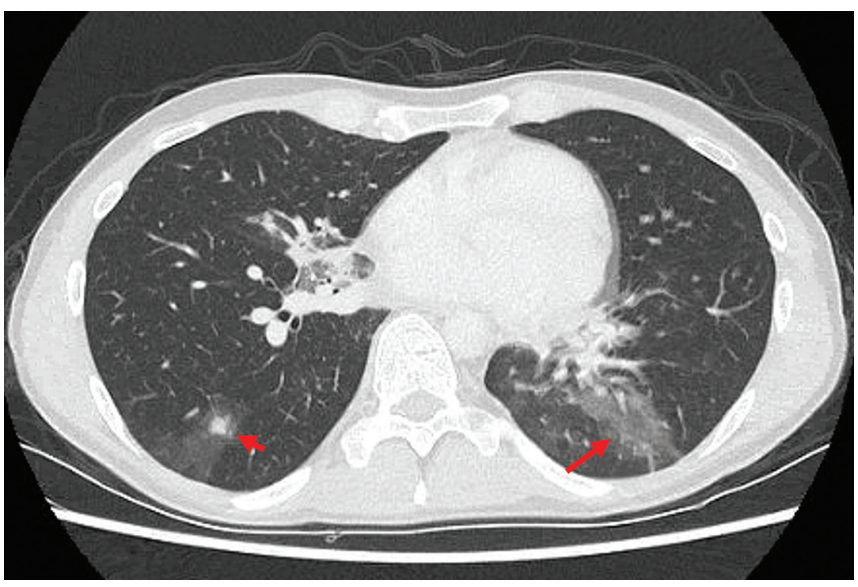

Figure 3: Computed tomography scan of the patient's chest showing a nodule with surrounding ground-glass opacity in the right lower lobe (short arrow) and consolidation in the left lower lobe (long arrow).

reported use of a nasal decongestant of natural sesame oil for the past 2 years to help with his sinusitis. Given this exposure history and consistency with the pathology results, we diagnosed exogenous lipoid pneumonia and counselled the patient to stop the antibiotics and oil products. When we reassessed him 3 months later, he reported that he was feeling better and less tired, but that he still had dyspnea on exertion. Pulmonary function tests showed improvements in his lung volumes, with a total lung capacity of $6.88 \mathrm{~L}(86 \%$ predicted), forced vital capacity of $4.31 \mathrm{~L}(70 \%$ predicted) and diffusion capacity of $87 \%$ predicted. 


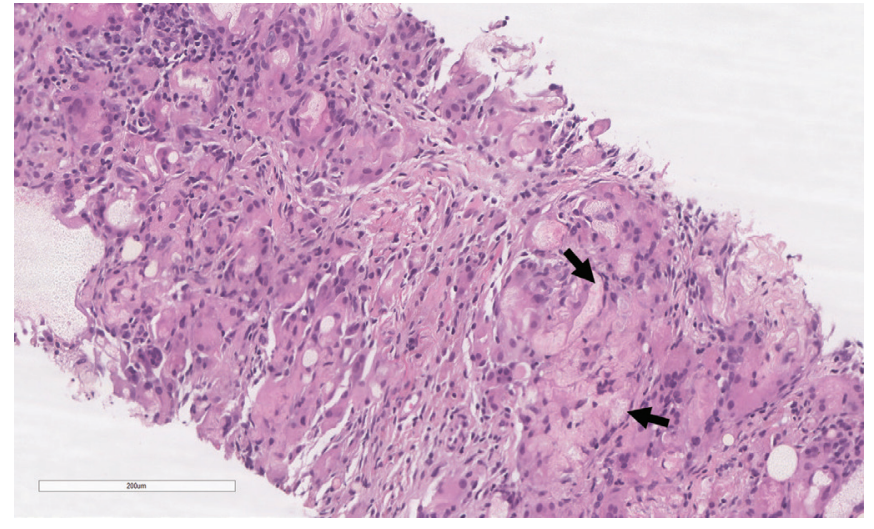

Figure 4: Lung biopsy pathology showing an amorphous, acellular and nonpolarizable substance with a foreign-body giant cell reaction. The amorphous substance is located inside intracytoplasmic vacuoles and appears as irregular sheets (arrows).

\section{Discussion}

We present a case of a 30-year-old man with nonresolving pneumonia. Besides radiographic findings, his blood work and physical examination were largely normal, leading to a diagnostic challenge and difficulty assessing treatment response. Our patient was initially suspected to have undertreated bacterial pneumonia caused by $P$. aeruginosa and S. marcescens that were not susceptible to clarithromycin and amoxicillin. However, subsequent treatment with ciprofloxacin should have provided adequate coverage for these organisms, assuming favourable susceptibility profiles. The history of $P$. aeruginosa and S. marcescens cultured from bronchoscopy in Poland is surprising as these are generally nosocomial respiratory infections. ${ }^{1,2}$ The patient did not have risk factors for communityacquired pneumonia with $P$. aeruginosa or other gram-negative bacteria, such as pulmonary disease or previous hospital admission, antibiotic use ${ }^{3}$ or oral steroid use.

Nonresolving pneumonia has been defined as an absence of clinical improvement, clinical worsening or absence of radiographic resolution within 12 weeks after adequate antibiotic therapy of a pulmonary infection with associated infiltrates. ${ }^{4}$ Patient characteristics associated with slowly resolving pneumonia include increased age, smoking, chronic illness, bacteremia, multilobar pneumonia and persistent fever or leukocytosis. ${ }^{5}$ Our patient, a young, relatively healthy nonsmoker, did not have these risk factors when he was initially treated for communityacquired pneumonia. Therefore, his prolonged course of illness was unexpected and required further investigation for alternative causes of his nonresolving pneumonia.

The differential diagnosis for nonresolving pneumonia includes difficult-to-treat infectious causes, as well as noninfectious causes, such as inflammatory and malignant diseases (Box 1). ${ }^{5,6}$ Given that the patient did not have any manifestations of extrapulmonary disease, did not have a history of cystic fibrosis or asthma, and did not have peripheral eosinophilia, leukocytosis or a history of pneumonitis-inducing drug exposure, many of these diagnoses were unlikely.

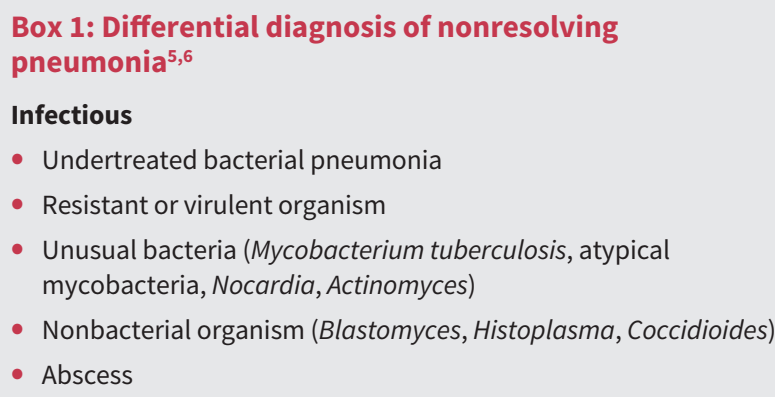

A thorough clinical history is key; had our patient's lipid exposures been identified earlier, he might have avoided a lung biopsy and some of his emergency department visits and hospitalizations. Bronchoscopy may be particularly beneficial to investigate a specific diagnosis in patients with nonresolving pneumonia who are younger than 55 years, nonsmokers, with multilobar pneumonia and longer duration of symptoms, ${ }^{7}$ which was the case in our patient. Although his second bronchoscopy was nondiagnostic, it ruled out many potential causes and showed clearance of $P$. aeruginosa and S. marcescens.

Lipoid pneumonia is a rare cause of nonresolving pneumonia. It is classified as endogenous (when caused by lipids from lung tissue broken down by obstructive or inflammatory processes) or exogenous (when caused by inhaled or aspirated oily substances) and may be further classified as an acute or chronic process. ${ }^{8}$ Acute exogenous lipoid pneumonia occurs with aspiration of a large quantity of material, and chronic exogenous lipoid pneumonia occurs with repeated episodes of aspiration or inhalation. ${ }^{8}$ There have been reports of exogenous lipoid pneumonia from aspiration or inhalation of sesame oil. ${ }^{9}$ On the basis of our patient's reported exposure history, he was likely chronically aspirating or inhaling flaxseed and sesame oil.

The pathophysiology of exogenous lipoid pneumonia involves the aspirated or inhaled fatty substance reaching the lung alveoli, where it is engulfed by macrophages. ${ }^{10}$ The macrophages eventually die and release the oil, resulting in inflammation, giant cell granulomatous reaction and fibrosis. ${ }^{10}$ The typical symptoms associated with acute exogenous lipoid pneumonia include 
cough, dyspnea and fever; chronic exogenous lipoid pneumonia may be asymptomatic or present with cough or dyspnea, and less commonly presents with fever, weight loss, chest pain and hemoptysis. ${ }^{8}$ Radiographic findings are variable. Acute exogenous lipoid pneumonia may present as bilateral segmental, lobar ground-glass or consolidative opacities in the middle and lower lobes. ${ }^{8}$ Computed tomography scans may show areas of fat density (i.e., low Hounsfield units); however, this may be obscured by superimposed inflammation. ${ }^{8}$ Chronic exogenous lipoid pneumonia presents similarly, but is more typically segmental in the lower lobes, with a peribronchovascular distribution. ${ }^{8}$ Oil red 0 staining may show lipids on bronchoalveolar lavage pathology. ${ }^{10}$ Treatment involves stopping use of the fatty substance; some case reports suggest that corticosteroids may also be helpful, depending on the severity of the pneumonia. Remission usually occurs within weeks in mild cases, but more severe cases may result in lung fibrosis and ongoing respiratory symptoms. ${ }^{9}$

Overall, our patient's history, symptoms and radiographic findings were consistent with exogenous lipoid pneumonia. He likely experienced a superimposed secondary bacterial infection. We have reported this adverse event in the context of use of a marketed product, sesame oil nasal spray, to the Natural Products Directorate of Health Canada.

\section{Conclusion}

Exogenous lipoid pneumonia is a rare cause of nonresolving pneumonia and may present with a superimposed bacterial infection. Our case highlights the importance of taking a detailed history that includes exposure to fatty or oily substances, particularly when inhaled or applied to the nasopharynx or oropharynx, as part of the work-up for nonresolving pneumonia.

\section{References}

1. Brzozowski M, Krukowska A, Galant K, et al. Genotypic characterisation and antimicrobial resistance of Pseudomonas aeruginosa strains isolated from patients of different hospitals and medical centres in Poland. BMC Infect Dis 2020;20:693.

2. Naumiuk $Ł$, Baraniak A, Gniadkowski M, et al. Molecular epidemiology of Serratia marcescens in two hospitals in Gdansk, Poland, over a 5-year period. Clin Microbiol 2004;42:3108-16 [published erratum in Clin Microbiol 2004;42:3914].

3. Arancibia F, Bauer TT, Ewig S, et al. Community-acquired pneumonia due to gram-negative bacteria and pseudomonas aeruginosa. Arch Intern Med 2002; 162:1849-58.

4. Low DE, Mazzulli T, Marrie T. Progressive and nonresolving pneumonia. Curr Opin Pulm Med 2005;11:247-52.

5. Johnson J. Slowly resolving and nonresolving pneumonias. Questions to ask when response is delayed. Postgrad Med 2000;108:15-122.

6. Kyprianou A, Hall CS, Shah R, et al. The challenge of nonresolving pneumonia: Knowing the norms of radiographic resolution is key. Postgrad Med 2003;113: 79-82.
7. Feinsilver SH, Fein AM, Niederman MS, et al. Utility of fiberoptic bronchoscopy in nonresolving pneumonia. Chest 1990;98:1322-6.

8. Betancourt SL, Martinez-Jimenez S, Rossi SE, et al. Lipoid pneumonia: spectrum of clinical and radiologic manifestations. AJR Am J Roentgenol 2010; 194:103-9.

9. Kuroyama M, Kagawa H, Kitada S, et al. Exogenous lipoid pneumonia caused by repeated sesame oil pulling: a report of two cases. BMC Pulm Med 2015; 15:135.

10. Hadda V, Khilnani GC. Lipoid pneumonia: an overview. Expert Rev Respir Med 2010;4:799-807.

\section{Competing interests: None declared.}

This article has been peer reviewed.

The authors have obtained patient consent.

Affiliations: Temerty Faculty of Medicine (Yeung, Rotin, Singh, Wu, Stanbrook), University of Toronto; Division of General Internal Medicine (Wu), University Health Network; Division of Respirology (Stanbrook), University Health Network, Toronto, Ont.

Contributors: All authors contributed substantially to the conception and design of the manuscript. Sabrina Yeung drafted the article. All authors revised it critically for important intellectual content, gave final approval of the version to be published and agreed to act as a guarantor of the work.

Content licence: This is an Open Access article distributed in accordance with the terms of the Creative Commons Attribution (CC BYNC-ND 4.0) licence, which permits use, distribution and reproduction in any medium, provided that the original publication is properly cited, the use is noncommercial (i.e., research or educational use), and no modifications or adaptations are made. See: https://creativecommons.org/licenses/by-nc-nd/4.0/

Acknowledgement: The authors thank Dr. Michael Cabanero of the University Health Network Department of Laboratory Medicine \& Pathobiology for providing the image of the lung biopsy histopathology and its interpretation.

Disclaimer: Matthew Stanbrook is a deputy editor for CMAJ and was not involved in the editorial decision-making process for this article.

Correspondence to: Matthew Stanbrook, matthew.stanbrook@uhn.ca

The section Cases presents brief case reports that convey clear, practical lessons. Preference is given to common presentations of important rare conditions, and important unusual presentations of common problems. Articles start with a case presentation (500 words maximum), and a discussion of the underlying condition follows (1000 words maximum). Visual elements (e.g., tables of the differential diagnosis, clinical features or diagnostic approach) are encouraged. Consent from patients for publication of their story is a necessity. See information for authors at www.cmaj.ca. 